Review Article

\title{
Current Bancroftian Filariasis Elimination on Thailand-Myanmar Border: Public Health Challenges toward Postgenomic MDA Evaluation
}

\author{
Adisak Bhumiratana, ${ }^{1}$ Apiradee Intarapuk, ${ }^{2}$ Surachart Koyadun, ${ }^{3}$ \\ Pannamas Maneekan, ${ }^{4}$ and Prapa Sorosjinda-Nunthawarasilp ${ }^{5}$ \\ ${ }^{1}$ Department of Parasitology and Entomology, Faculty of Public Health, Mahidol University, 420/1 Rajvithi Road, Rajthewee, \\ Bangkok 10400, Thailand \\ ${ }^{2}$ Department of Clinic, Faculty of Veterinary Medicine, Mahanakorn University of Technology, 140 Cheum-Sampan Road, \\ Nong-Chok, Bangkok 10530, Thailand \\ ${ }^{3}$ Department of Disease Control, Ministry of Public Health, Office of Disease Prevention and Control 11, \\ Nakhon Si Thammarat 80000, Thailand \\ ${ }^{4}$ Department of Tropical Hygiene, Faculty of Tropical Medicine, Mahidol University, Bangkok 10400, Thailand \\ ${ }^{5}$ Department of Fundamentals of Public Health, Faculty of Public Health, Burapha University, Chonburi 20131, Thailand
}

Correspondence should be addressed to Adisak Bhumiratana; adisak.bhu@mahidol.ac.th

Received 25 December 2012; Accepted 6 February 2013

Academic Editors: P. Horrocks, W. Mosca, P. A. Nogueira, J. Ogwal-Okeng, and S. Tesana

Copyright (C) 2013 Adisak Bhumiratana et al. This is an open access article distributed under the Creative Commons Attribution License, which permits unrestricted use, distribution, and reproduction in any medium, provided the original work is properly cited.

\begin{abstract}
From regional and global perspectives, Thailand has progressed toward lymphatic filariasis transmission-free zone in almost entire endemic provinces, being verified by WHO by the end of 2012 after the 5-year implementation of mass drug administration (MDA) with diethylcarbamazine and albendazole as part of the National Program to Eliminate Lymphatic Filariasis (PELF) (2002-2006) and a 4-year expansion of post-MDA surveillance (2007-2010). However, Thai PELF has been challenging sensitive situations of not only border crossings of local people on Thailand-Myanmar border where focal distribution of forest- and forest fringe-related border bancroftian filariasis (BBF) is caused by nocturnally subperiodic Wuchereria bancrofti in local people living in pockets of endemic villages, but also intense cross-border migrations of Mon and Tanintharyi workers from Myanmar to Thailand who harbor nocturnally periodic $W$. bancrofti microfilaremic infection causing the emergence of imported bancroftian filariasis (IBF). Thus, this paper discusses the apparent issues and problems pertaining to epidemiological surveillance and postgenomic MDA evaluation for 2010-2020 convalescent BBF and IBF. In particular, the population migration linked to fitness of benzimidazole-resistant $W$. bancrofti population is a topic of interest in this region whether the resistance is associated with pressure of the MDA 2 drugs and the vulnerabilities epidemiologically observed in complex BBF or IBF settings.
\end{abstract}

\section{Global and Regional Perspectives on Lymphatic Filariasis Elimination}

Life-threatening lymphatic filariasis (LF) is a mosquito-borne parasitic disease caused by two main filarial nematodes: Wuchereria bancrofti and Brugia malayi, and to a very lesser extent by Brugia timori. The disease affects about 1.3 billion people in 81 countries and territories in Asia-Pacific, Africa, and Americas; of these, estimated 120 million people are infected (Figure 1) [1]. It is estimated that about 600 million people live in endemic countries in South and East Asia (SEA) region accounting for $60 \%$ of global figure. Approximately, 60 million SEA people harboring microfilaremic infections account for a half of globally active LF burden [2]. The infection with either of these parasites in an endemic population can be eliminated as the result of large-scale control, that is, reducing the infection prevalence to the level below transmission threshold or to be considered as no longer public health 


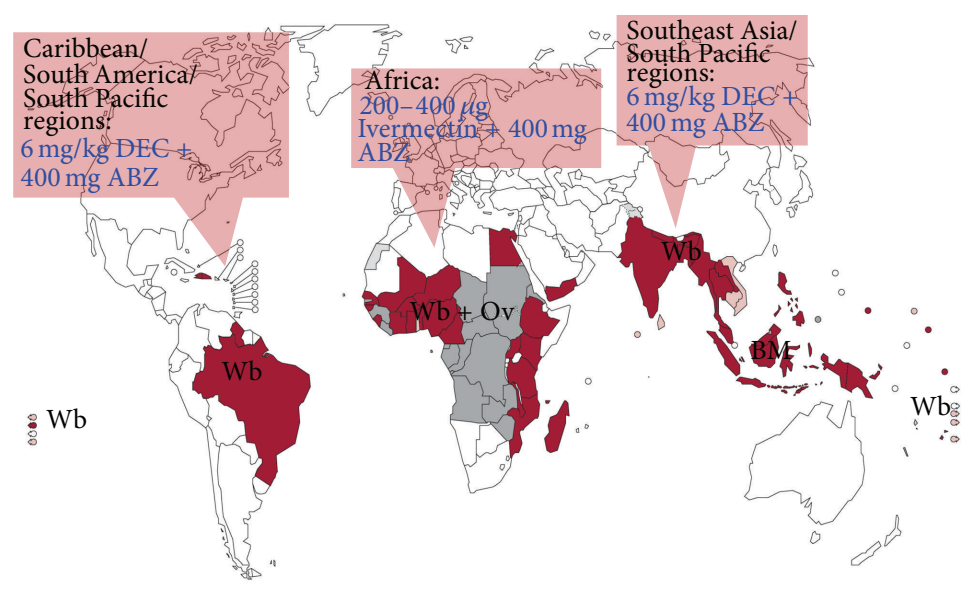

Endemic countries and territories implementing preventive chemotherapy
Endemic countries and territories where the target was achieved and
implementation stopped
$\square$ Endemic countries and territories not started implementing preventive
chemotherapy
$\square$ Nonendemic countries and territories
$\square$ Not applicable

FIGURE 1: Current status of endemic countries and territories implementing MDA 2 drugs, 2011. The GPELF focuses on geographically associated coverage of MDA 2 drugs to the target population $\geq 2$ years of age in transmission areas in endemic countries and territories. ABZ: albendazole, Bm: Brugia malayi, DEC: diethylcarbamazine, Ov: Onchocerca volvulus, and Wb: Wuchereria bancrofti. Map was adapted from the global health observatory map gallery available at http:/gamapserver.who.int/mapLibrary/app/searchResults.aspx.

problem. Thus, this potentially eradicable disease has been addressed as public health problem worldwide as the result of medically and epidemiologically advanced tools used on a large scale in surveillance and control [3-7]. As recommended by the World Health Organization (WHO), two pillars of global elimination strategies emphasize interruption of transmission and elimination of the infection in humans among target population (i.e., the population at risk of, or affected with, the infection in a transmission area) and the other large scale morbidity control to prevent disease and disability [3-7]. To achieve this ultimate goal, the Global Program to Eliminate Lymphatic Filariasis (GPELF) predetermines an annual mass drug administration (MDA) using a single-dose combination of antifilarial and anthelmintic drugs that serves as preventive chemotherapy and transmission control (PCT) [6]. The PCT with MDA 2-drug regimens include $6 \mathrm{mg} / \mathrm{kg}$ diethylcarbamazine citrate (DEC) plus $400 \mathrm{mg}$ albendazole (in $W$. bancrofti transmission areas where Onchocerca volvulus is not coendemic), or with 200$400 \mu \mathrm{g}$ ivermectin plus $400 \mathrm{mg}$ albendazole (in W. bancrofti transmission areas where $O$. volvulus is coendemic) (Figure 1) [2-7]. An MDA 2-drug regimen is given once a year for $4-6$ years to an eligible target population $\geq 2$ years of age. This regime has been considered an effective elimination strategy as part of the National Program to Eliminate Lymphatic Filariasis (PELF) in all endemic countries allied to GPELF. Continuation of annual MDA 2 drugs has effects on primarily reducing microfilarial density (i.e., the amount of microfilariae (Mf) circulating in peripheral blood in regard to their periodicity) in an infected individual and hence dramatically reducing microfilaremia prevalence (i.e., a number of microfilaremic persons determined by Giemsastained standard thick blood film in a total target population) in an affected community. Approximately, 60-80\% coverage of an annual MDA 2-drug regimen (i.e., an annual number of compliant persons treated with MDA 2 drugs in an entire eligible target population in a transmission area as implementation unit) for 4-5 consecutive years is considered effective enough to interrupt transmission in geographically defined control areas in the absence of vector control [5]. Therefore, the scaling-up coverage with geographically associated MDA 2 drugs (i.e., a number of implementation units with annual MDA 2 drugs in a total number of implementation units) can reduce infection/disease prevalence (i.e., annually cumulative number of microfilaremic persons and/or acute LF cases), thus preventing new infection, within an endemic country and, internationally, within the endemic countries and territories [6-9].

In this regard, geographical coverage of annual MDA 2 drugs in an almost entirely at-risk population can reach up to the level of community effect such that the infection prevalence (i.e., microfilaremia rate for $W$. bancrofti or $B$. malayi, antigenemia rate for $W$. bancrofti only, and filarial IgG4 antibody-positive rate for $B$. malayi only) can be domestically arrested after several rounds of $\mathrm{MDA}[3,6-$ 12]. A marked reduction of infection prevalence in almost 
all transmission areas with MDA 2-drug coverage can reach to a nearly zero-ground infection level within a timeline of completely planned MDA rounds, or even a prolonged period of annual MDA 2 drugs, and post-MDA evaluation. If being the case, the PELF can achieve the target of the elimination of LF infections in humans. Undoubtedly, LF elimination efforts with chemotherapeutical uses transcend knowledge of biomedical science in interactions of parasites, vectors, and human hosts. Indeed, global challenges to cope with this no longer deviating disease rely primarily on the availability of existing and alleviating control measures and the reduction of weakness or constraints in LF elimination management at country level. In the optimistic view of the GPELF, if collectively effective management solutions of the PELFs demonstrate most notable successes, the achievable goal of LF elimination in 2020 would reflect the eradication of global LF burden that appears to be worldwide zero-ground incidence within the geographic regions $[3,6-9]$.

\section{Progress toward Lymphatic Filariasis Elimination in Thailand}

Thailand is known for low endemicity of LF, which has focal distribution of nocturnally subperiodic $W$. bancrofti along Thailand-Myanmar border [13-15] and, concurrently, both nocturnally subperiodic and periodic B. malayi in the South [16]. Meanwhile, nocturnally periodic $W$. bancrofti does not exist in Thailand but appears to be highly prevalent in Myanmar [2,9] and commonly found in microfilaremic Myanmar migrant workers in Thailand [15]. Due to intense movements of cross-border Myanmar migrant workers (MMWs) to Thailand, many receptive urban and suburban areas in which Culex quinquefasciatus can infest are at risk of introduced transmission of this parasite [17-19]. Similar to other endemic countries implementing MDA 2 drugs (DEC plus albendazole) in the SEA region, Thai PELF emphasizes a 5-year-long MDA 2-drug implementation in transmission areas countrywide (Figure 2). With these complex epidemiologic figures, Thai PELF management by fiscal years 2002-2006 deliberately performs with two strategic approaches through which the administrative levels of existing national public health system and healthcare services can implement LF management strategies suited to LF burdens in different provinces. The first-line approach to interrupt both $W$. bancrofti and $B$. malayi transmission in transmission areas employs not only a free distribution of MDA 2 drugs to local Thai target population but also an increase in coverage of annual MDA 2 drugs (Figure 2(A)). Accordingly, the second-line approach to prevent introduced transmission of nocturnally periodic $W$. bancrofti utilizes a large-scale MDA with multiple-dose $\mathrm{DEC}$ at provincial level to all eligible foreign migrant workers, for example, primarily MMWs (Figure 2(B)).

In fact, Thai PELF corroborates the existing filaria control program that is radically integrated with the national control program for vector-borne diseases. A vertical 40-year-long filaria control program has focused mainly on the reduction in microfilaremia prevalence in transmission areas for $W$. bancrofti and $B$. malayi through which the surveillance and control are augmented using a combination of prevention/control measures, which are active case detection by night blood survey, single-dose administration with $6 \mathrm{mg} / \mathrm{kg}$ DEC repeated at 6-month intervals for 2 consecutive years for an individual infected, foci investigation, vector control, LF rehabilitation, and health education. The effective implementation of the national filaria control program that had continually projected a decline of microfilaremia prevalence (Figure 3) is the prototype of Thai PELF that has been performed on a large scale with the adoption of MDA 2 drugs since 2002. As a result, a microfilaremia rate of $3.2 \%$ in the beginning year 1961 of filaria control dramatically declined to a marginal level of $0.1 \%$ microfilaremia rate for few years 20012002 before the start of Thai PELF as a pre-MDA baseline microfilaremia prevalence (Figure 3(a)). Endemic locality of LF has been restrained focally within 336 villages in 11 endemic provinces: Mae Hong Son, Chiangmai, Lumphoon, Tak, Kanchanaburi, Ratchaburi, Ranong, Suratthani, Krabi, Nakhon Si Thammarat, and Narathiwat (Figure 3(b)). All 336 endemic villages were designated as implementation units (IUs) of MDA 2 drugs (DEC plus albendazole). By the end of 2004, mapping of LF distribution was completed. A total of 150 thousand people ( $\sim .2 \%$ of total population) living in the entire transmission areas were $100 \%$ covered with MDA 2 drugs (Figure 3(b)). With regard to the regional SEA target, Thailand would appear to be LF transmission-free by the end of 2010 [2]. A progress toward LF elimination in 2010 demonstrates that Thai PELF has terminated domestically the infections with $W$. bancrofti and B. malayi in almost the entire target population in 11 implementing provinces except Narathiwat after a 5-year implementation of the PELF (20022006) and a 4-year expansion of post-MDA surveillance for LF (2007-2010) [2,9]. Regarding the achievement of Thai PELF, it will be a crucial step for the preparation of LF-free zone being notified by WHO by the end of 2012 . Meanwhile, Narathiwat will continue of MDA in B. malayi transmission areas because there are several counterintuitive problems including inadequate health personnel, low MDA 2-drug coverage, and limited control of the zoonotic B. malay $i$ infection in domestic cats. Moreover, there have been a line of evidence that if Thai PELF completely terminates annual MDA 2 drugs in transmission areas on or close to ThailandMyanmar border [15, 17-21], the recrudescence of W. bancrofti transmission will occur due either to border crossings of local border people between Thailand and Myanmar or to cross-border migrations of MMWs to Thailand [22]. Concurrently, these phenomena are thought to be epidemiologically linked with human-vector combinations in these vulnerable people, and routine surveillance can detect resurgence of $W$. bancrofti transmission caused by these anomalies timely if there is enough empiric data/information to understand the vulnerabilities in how contagious is the parasite in such complex epidemiological settings.

\section{Border Bancroftian Filariasis Settings and Dynamics}

Border bancroftian filariasis (BBF) is forest- and forest fringe-related bancroftian filariasis caused by nocturnally 


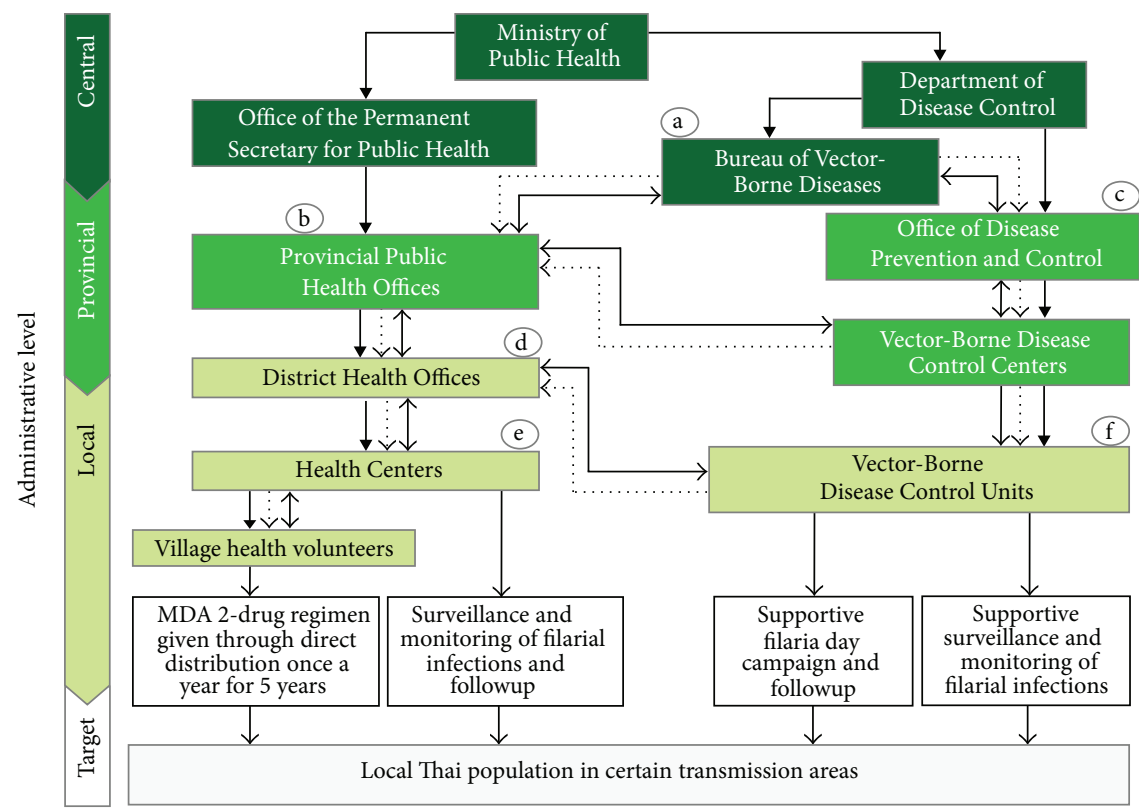

(A)

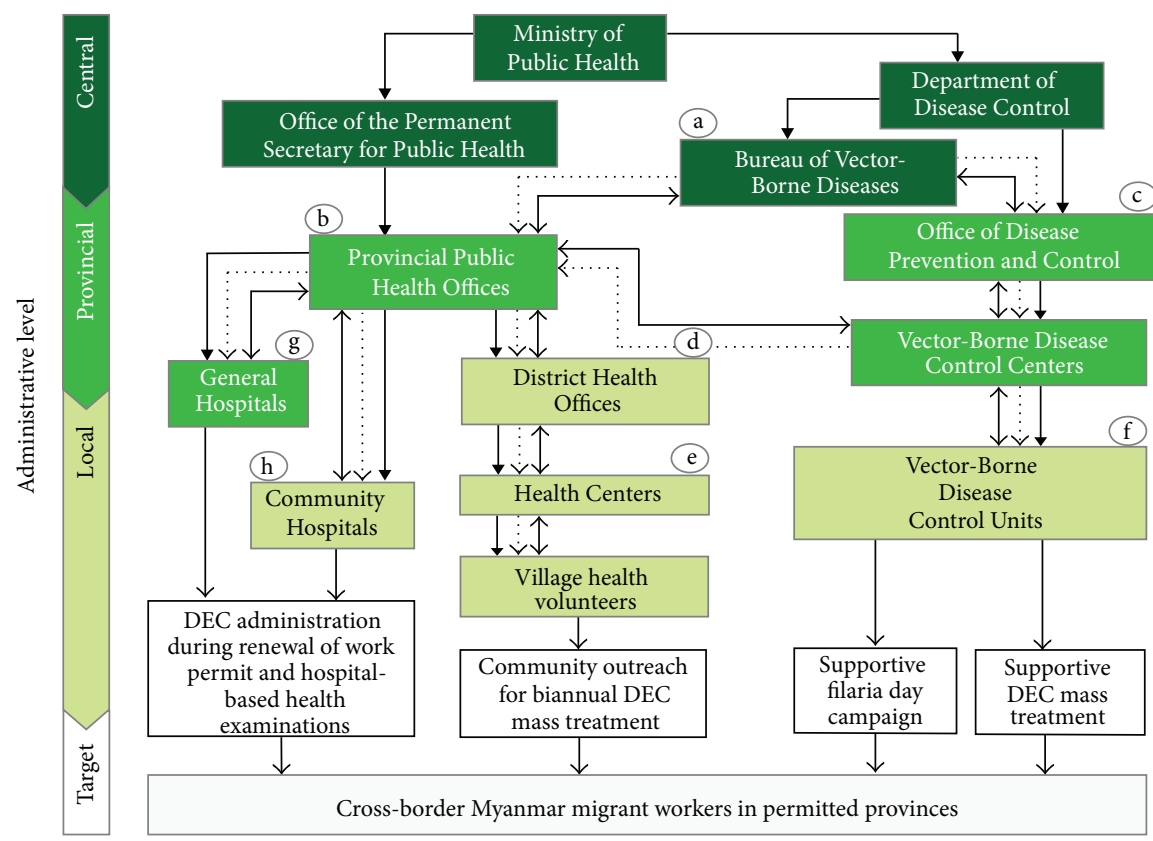

(B)

FIGURE 2: Strategic approaches to lymphatic filariasis elimination used in the National Program to Eliminate Lymphatic Filariasis (Thai PELF). (A) MDA 2-drug regimen ( $6 \mathrm{mg} / \mathrm{kg}$ DEC plus $400 \mathrm{mg}$ albendazole) is given once a year for 5 consecutive years to local Thai populations with $\geq 2$ years of age in certain transmission areas. This first strategic approach is dependent on interrupting transmission and hence eliminating human infections. (B) Multiple-dose DEC that makes use of DEC administration during renewal of work permit and hospital-based heath examination, community outreach for biannual DEC mass treatment, and supportive DEC treatment via malaria clinics or mobile clinics. For both strategic approaches, PELF management mechanisms involve direction and command $(\rightarrow)$, coordination (i.e., managerial, financial, and technical) $(-\rightarrow)$, data flow and integration $(\uparrow)$, and elimination strategy operation $(\downarrow)$ ). The implementers at different administrative levels (i.e., central, provincial, and local) of existing national public health system are involved in strategic deployment: a - Thai PELF program manager; $b$ and c-PELF program coordinators at provincial level; $d$ and e-implementers in charge of the implementation of MDA 2-drug distribution to Thai target population, surveillance and monitoring of filarial infections, and followup as well as of biannual DEC mass treatment to Myanmar migrant workers; $\mathrm{f}$-implementers in charge of supportive PELF activities of Filaria day campaign, followup, and surveillance and monitoring of filarial infections as well as supportive DEC treatment via malaria clinics or mobile clinics; $g$ and $h$-implementers providing not only a single-dose administration with $300 \mathrm{mg}$ DEC recommended by the PELF as provocative-day test to assess filarial infections but also a $400 \mathrm{mg}$ albendazole recommended by migrant worker health service program to treat geohelminth infections. 


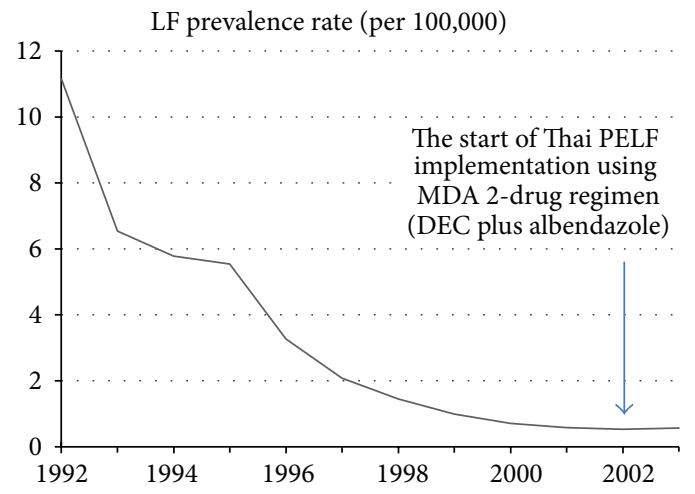

(a)

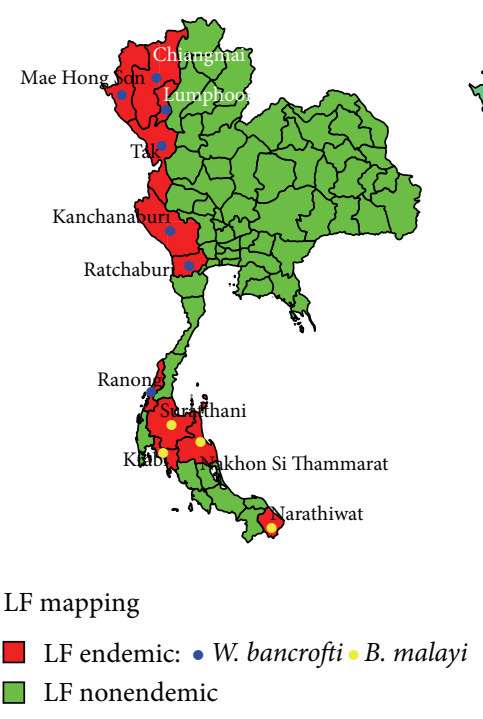

(b)

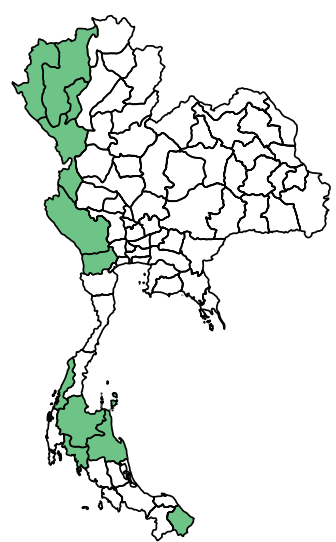

MDA IU coverage

Endemic IUs $100 \%$

Nonendemic

FIGURE 3: Current LF situation and the geographically associated 11-province targets of the implementation of the PELF in Thailand. (a) A dramatic decline of LF prevalence rates (per 100,000 population), 1992-2002. (b) In each endemic province, the pocket villages that are identified as transmission areas (TA) or the implementation units (IUs) of mass drug administration are targeted by the PELF. The $100 \%$ of MDA IU coverage was reported as of 2003. Data were adapted from the Bureau of Vector-borne Diseases, Department of Disease Control, Ministry of Public Health, Thailand.

subperiodic $W$. bancrofti on the Thai-Myanmar border (Figure 4). The infection occurs with the acquisition of naturally mosquito-borne transmission in hilly endemic villages, which are mostly confined to Thai-Myanmar border provinces including Tak, Mae Hong Son, and Kanchanaburi. $\mathrm{BBF}$ appears to be low endemic but relatively coendemic with malaria. With regard to in vivo susceptibility to nocturnally subperiodic $W$. bancrofti, a diverse group of anopheline and culicine mosquitoes is thought to be natural vector [23]. Two common malaria vectors such as Anopheles maculatus and An. minimus are more likely to play significant role in experimentally induced infection and infectivity than Aedes niveus, Ae. desmotes, An. dirus, Culex quinquefasciatus, and Mansonia uniformis. However, potential transmission of these vectors in transmission areas remains unclear. For BBF epidemiology, the cumulative infection prevalence of $\mathrm{BBF}$ normally increases with increasing ages but appears to be gender-specific; microfilaremic male patients are about 2-fold higher than female patients $[13,14]$. The prevalence is lower in endemic population aged $\geq 50$ years for both genders [14]. Adult patients older than 30 years develop hydrocele as commonly characterized as chronic lymphatic filariasis in $\mathrm{BBF}$ [14]. However, there is the likelihood that vulnerable local border people between Thailand and Myanmar have frequent exposure of human-vector combinations (Figure 4). Most likely explanation is the underlying human movement-a key behavioral factor that can envisage the emergence or recrudescence of BBF among endemic local border populations in hotspots, that is, endemic localities geographically associated with a cyclic transmission of nocturnally subperiodic $W$. bancrofti in which uncertain transmission possibly occurs with ecological changes of a plethora of mosquito vectors and demographic changes of local border populations between Thailand and Myanmar. Such human movements have created transmission dynamic of BBF in a hotspot area of Tak border province, Thailand [14]. Regardless of human movements in the pocket of endemic villages on Thai-Myanmar border, it was likely to show high overall rates of infection and disease prevalences: $3.7 \%$ microfilaremia, $23.7 \%$ antigenemia, and $4.6 \%$ male hydrocele. However, when examined for the relationship between human movements and infection or disease prevalence, it was more likely to show a significant LF burden in migratory persons from Myanmar residing in the villages. Migratory persons had 5.8\% microfilaremia, $32.5 \%$ antigenemia, and $8.3 \%$ male hydrocele, comparing to local persons that had only $1 \%$ microfilaremia and $13.1 \%$ antigenemia. Although nearly $1 \%$ microfilaremic infection prevalence observed in some endemic villages seems to be as relatively low as a transmission threshold, it does not mean that local transmission is stable in $W$. bancrofti-endemic populations in hotspots on Thai-Myanmar border. Human movements and activities are likely to be related to male adults who contribute to the extent of the infection intensity on Thai-Myanmar border. For instance, $W$. bancrofti infection intensity in local endemic border population of Tak province showed a significant median antigen load in antigenemic male persons 3-fold higher than that observed in antigenemic female persons [15]. Also, there was significant difference in antigen loads among age groups, but there was no correlation between antigen loads and ages. With regard to microfilaremic status, antigenemic persons with microfilaremia had also a significant median antigen load 4-fold higher than that observed in antigenemic persons with amicrofilaremia [15]. Clearly, annual MDA 2 drugs have effects on the reduction of 
microfilaremia prevalence in $\mathrm{BBF}$ but relatively have longer time to decrease antigenemia prevalence. It is, however, unwise to suggest that transmission of $W$. bancrofti is stable on the border because transmission dynamic of BBF is likely to be associated with the cross-border people uncovered by annual MDA 2 drugs in Myanmar (Figure 4). Moreover, little is known about protective actions the people comprehend and practice routinely and preventive measures the people can afford. Thus, complex BBF settings have been challenging the effectiveness of Thai/Myanmar PELF implementation in several ways.

Although the accurate infection intensity of $W$. bancrofti in both local border people and migratory people on ThaiMyanmar border remains to be established, it is likely that there is a marked reduction of microfilaremia prevalence in the sentinel sites on Thai-Myanmar border as the result of 2-3 rounds of MDA. Nearly zero-ground microfilaremia prevalence was estimated up to the final round-up in 2006 of stopping MDA 2 drugs. Nonetheless, there is need for the continuation of surveillance and monitoring of the infection although there is the termination of Thai PELF on the border. It is possible that a large number of local Myanmar border people living in transmission areas close to the border provinces of Thailand were not being treated with MDA 2 drugs. Due to the absence of vector control in the PELF, transmission dynamic of BBF in local border people has, therefore, been created upon the interconnections underlying border crossings, human settlements with per se breeding sites and forest activities, behavioral risks, and discontinuing treatment (Figure 4). Moreover, for the Thai/Myanmar PELF, these factors might influence the national geographical coverage of PELF implementation on the border if there are low MDA 2-drug compliance of the eligible target population on the border and demographic changes of the border populations. With these underlying vulnerabilities, BBF would appear to be an emerging problem unless there is epidemiological and entomological surveillance for 20102020 convalescent BBF. This information might support the need for LF management strategies targeting complex BBF. The most likely explanation is that border crossings of local border people on Thai-Myanmar border may have influenced geographical coverage of Myanmar PELF rather than Thai PELF, and, consequently, a prolonged timeline of planned MDA 2 drugs is expected as underlying the infection intensity of $\mathrm{BBF}$ along the border as required to clear the infections among the cross-border people. Strategic approaches to BBF are to enhance and strengthen corroboration of local health authorities and networks, particularly in vulnerable areas where health service units such as malaria posts can approach community outreach for distribution of MDA 2 drugs, and more importantly, to establish epidemiological and entomological surveillance and monitoring of the MDA 2 drugs' impacts on the $W$. bancrofti population.

\section{Imported Bancroftian Filariasis Settings and Dynamics}

Only the $W$. bancrofti nocturnally periodic phenotype exists in Myanmar and the main vector is CX. quinquefasciatus. A national mosquito control program that has been launched since 1998 is to reduce morbidity, namely, interrupting transmission and treating infected persons. Soon after pilot and successive trials of annual MDA with single-dose DEC in highly endemic areas, Myanmar PELF was planned to cover target population living in endemic districts since 2001. By the end of 2004, it was estimated about 53.4 million of population at risk of LF ( $~ 95 \%$ of total population) in Myanmar living in 63 districts: 39 endemic, 6 nonendemic, and 18 uncertain, which were designated as IUs for MDA 2-drug coverage with the same DEC plus albendazole (Figures 5(a) and 5(b)) $[2,9]$. The complete mapping of LF distribution covered 153 out of a total of 324 endemic townships, 22 nonendemic, and 149 uncertain. The pre-MDA baseline microfilaremia prevalence varied between 1 and $7 \%$ and the Mf density ranged between 115 and $675 \mathrm{Mf} / \mathrm{mL}$. The MDA IUs coverage $>80 \%$ was implemented only in 22 out of 39 endemic IUs covering a target population of 17.9 million in Magway (3.9), Sagaing (4.6), Mandalay (6.4), Rakhine (2.9), and Chin (0.1) (Figure 5(c)). After 2 rounds of the MDA, positive microfilaremia rates were estimated: Magway 0.6-3.6\%, Sagaing 0.2-6.6\%, Mandalay 7.3\%, Rakhine 0.2$12.6 \%$, and Chin $1.4-1.6 \%$. The national geographical MDA coverage, on the other hand, was $40 \%$ of the entire endemic MDA IUs. Most markedly, a demographic stochasticity and a depopulation in rural-urban gradient may account for a metapopulation of the eligible populations living in the MDA IUs not covered by Myanmar PELF during the years 20012004.

Myanmar is a country of origin of human migration to Thailand. Of the total at-risk population of Yangon Division (3.1 million), Mon State (2.6 million), and Tanintharyi Division (1.4 million) (Figure 5(c)), this could result in extensive movements of large numbers of MMWs from these 3 endemic regions that they voyaged either seasonally or periodically to Thailand for labor forces in both the agriculture and industry [17-21, 25]. Their internal migration in Myanmar has been largely unknown, but intense migrations of cross-border MMWs to Thailand are involved in chain migration of Mon and Tanintharyi migrant workers due to "Push and Pull" effects (Figures 6 and 7). Other than Laos and Cambodian workers, MMWs have recently been identified as microfilaremic carriers causing the emergence of imported bancroftian filariasis (IBF) in Thailand [17-20]. IBF is caused by nocturnally periodic $W$. bancrofti that can be transmitted by $C x$. quinquefasciatus potent vector. This vector also is commonly found in urban and suburban areas in Thailand as a predominant species to endemic MDA IUs in Myanmar [2,9]. The vulnerability that may favor an introduced transmission of nocturnally periodic $W$. bancroft $i$ occurs with a number of microfilaremic persons in at-risk population with low compliance of MDA 2 drugs $(300 \mathrm{mg}$ DEC plus $400 \mathrm{mg}$ albendazole) in a hotspot village on the Thai-Myanmar border or in low-hygienic human settlements with per se breeding Cx. quinquefasciatus. More detailed data/information of potential transmission of $C x$. quinquefasciatus in either vulnerable areas of Thailand or endemic settings of Myanmar is required. However, if behavioral and occupational risks exist, MMWs seem to have more 

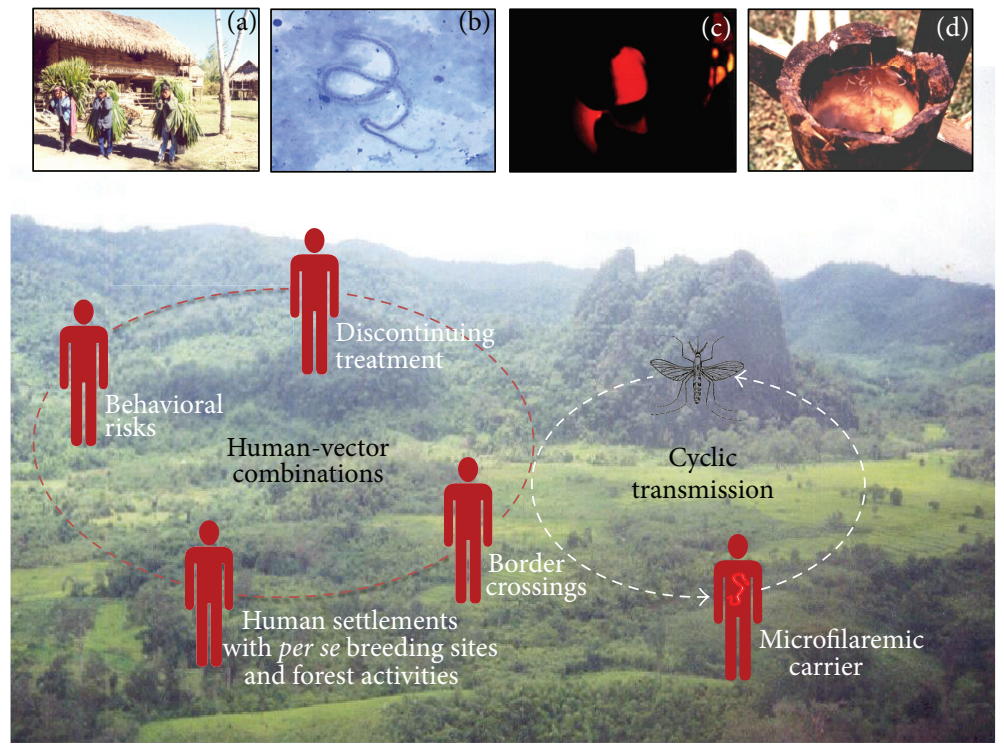

Figure 4: Border bancroftian filariasis settings and dynamics. A cyclic transmission of nocturnally subperiodic W. bancrofti in an endemic population in a pocket village on the Thai-Myanmar border (a) occurs with a number of microfilaremic carriers (b), a less number of cases with chronic lymphatic pathology, commonly hydrocele in adult males older than 30 years (c), and a density of potent Aedes niveus vector in transmission focus (d). However, its transmission dynamic is dependent on human-vector combinations underlying border crossings, human settlements with per se breeding sites and forest activities, behavioral risks, and discontinuing treatment.

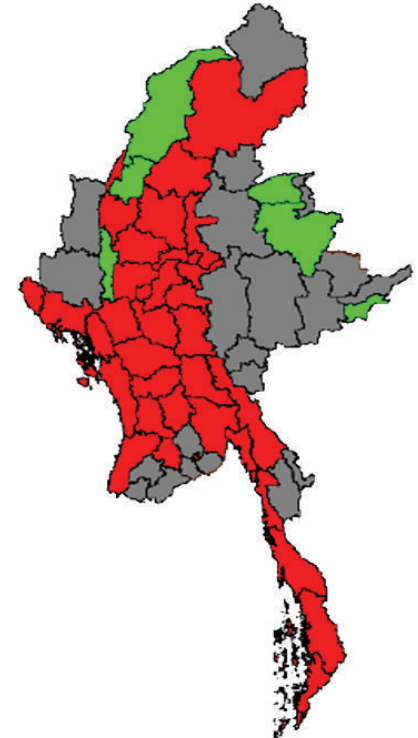

LF mapping

$\square$ LF endemic

LF nonendemic

Uncertain areas

(a)

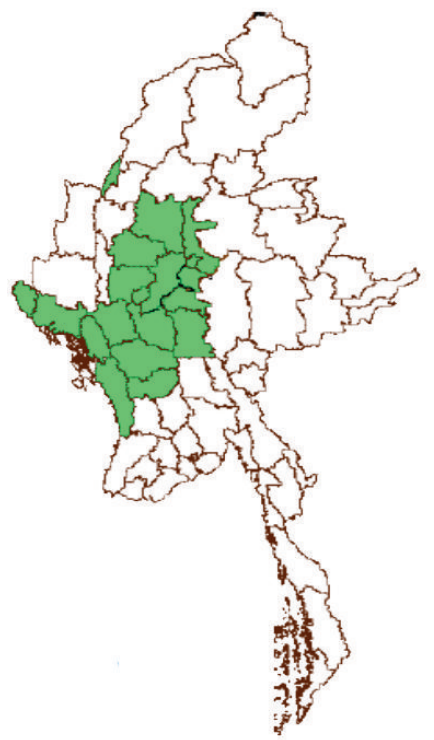

MDA IU coverage

Endemic IUs $>80 \%$

Endemic IUs not covered/ nonendemic/uncertain

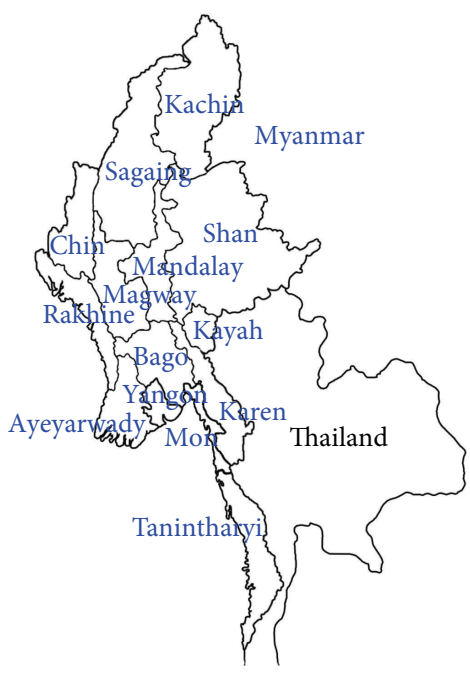

(b)

(c)

Figure 5: Myanmar PELF implementation as of 2004. (a) Mapping of LF distribution with district boundary. Sixty-three endemic districts designated as implementation units (IUs). (b) MDA coverage for the only 22 out of 39 endemic IUs. Data were obtained from the electronic file (http://209.61.208.233/LinkFiles/New_Lymphatic_Filariasis_Annual_Report_Myanmar_2004.pdf), deposited at the website http://www.searo.who.int/en/. (c) Map of Myanmar having 14 administrative divisions and states. 


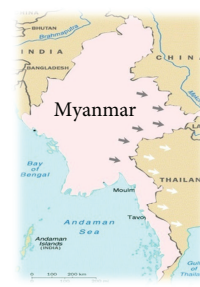

(a)

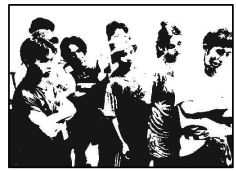

(b)

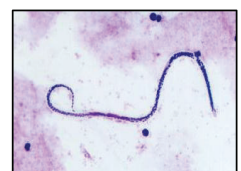

(c)

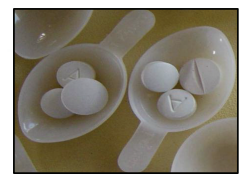

(d)

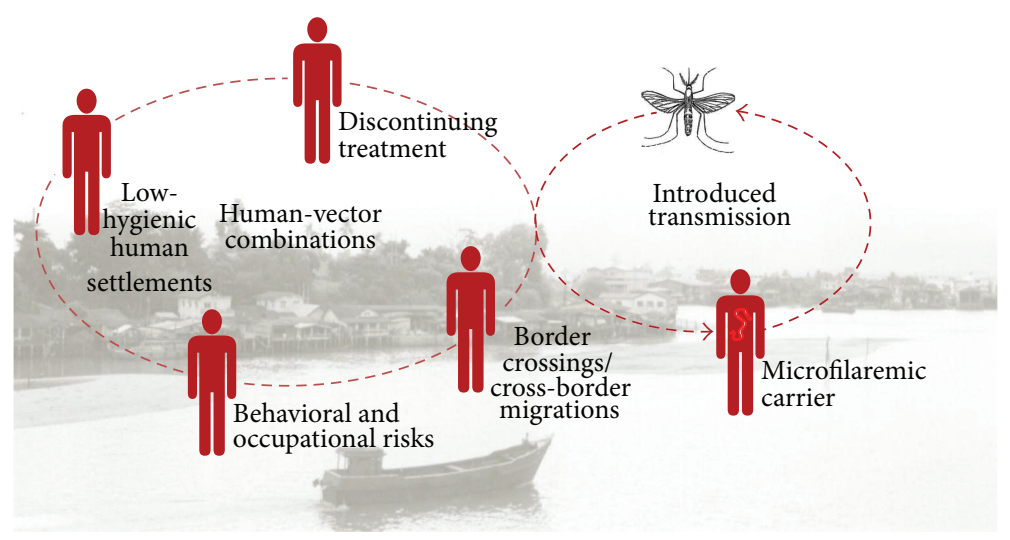

FIGURE 6: Imported bancroftian filariasis settings and dynamics. As a result of cross-border migrations of Myanmar migrant workers (MMWs) to Thailand (b) influenced by "Push and Pull" effect (a), an introduced transmission of nocturnally periodic W. bancrofti in at-risk population in a hotspot village on the Thai-Myanmar border or in urbanized areas occurs with a large number of MMWs including microfilaremic carriers (c) uncovered by the multiple-dose DEC treatment or MDA 2 drugs (300 mg DEC plus $400 \mathrm{mg}$ albendazole) (d). However, it is likely that more frequent human-vector combinations underlying border crossings and cross-border migrations, behavioral and occupational risks, low-hygienic human settlements, and discontinuing treatment will create a transmission dynamic in transmission-prone area where breeding Cx. quinquefasciatus.

frequent human-vector contacts and this risk will accelerate the introduced transmission in transmission-prone areas of Thailand.

The epidemiology of IBF remains to be established because it is very difficult to determine accurate infection prevalence and intensity. However, several reports showed the likelihood of varying infection rates, that is, either with microfilaremia, antigenemia, or filarial IgG4 antibody observed in different settings over time periods $[15,17-22$, 26]. For instance, $W$. bancrofti infection intensity in MMWs showed a significant median antigen load in antigenemic female persons 3-fold higher than that observed in antigenemic male persons but did not appear to be related with age groups [15]. There was a marked difference in antigen loads observed in MMWs 3-fold less than that observed in local border people. Regarding the microfilaremic infection status, antigenemic persons with microfilaremia had also a significant median antigen load 9-fold higher than that observed in antigenemic persons with amicrofilaremia [15]. However, microfilaremia rates are estimated from less than $0.1 \%$ to $\sim 1 \%$, but a diverse range of antigenemia rates is likely seen. On the other hand, Myanmar migrant workers with microfilaremia can be observed among those with short-term migration more than long-term migration [19], but it is very difficult to determine whether a marginal microfilaremia rate may account for introduced transmission in transmission-prone areas. This information might also reflect the needs for LF management strategies of integrated Thai-Myanmar PELFs applied to cross-border MMWs that may have longer timelines of planned MDA 2 drugs underlying the persistence of IBF if required to clear the infections in cross-border MMWs. More interestingly, there is also a need for epidemiological and entomological surveillance and monitoring of the MDA 2 drugs' impacts on the $W$. bancrofti population.

\section{Chain Migration Model of MMWs and Challenges to Thai/Myanmar PELF}

In large-scale transmission control of the IBF, Thai PELF targeting dynamic MMWs makes use of multiple-dose MDA with $300 \mathrm{mg}$ DEC regimens: DEC administration during the renewal of work permit and hospital-based health examinations, community outreach for biannual DEC mass treatment, and supportive DEC treatment via malaria clinics and mobile clinics (Figure 2(B)) [17]. In addition to what is recommended by $\mathrm{WHO}$, the implementation of this multipledose MDA with $300 \mathrm{mg}$ DEC has been proposed as aptly effective as for IBF control efforts in Thailand. This strategy optionally suited to cover those migrants who may access to health settings in different provinces is thought to have effects on reduction of microfilaremia and antigenemia prevalences in MMWs [17-19, 21]. During a renewal of work permit and hospital-based health examinations, any enrollee is given a 


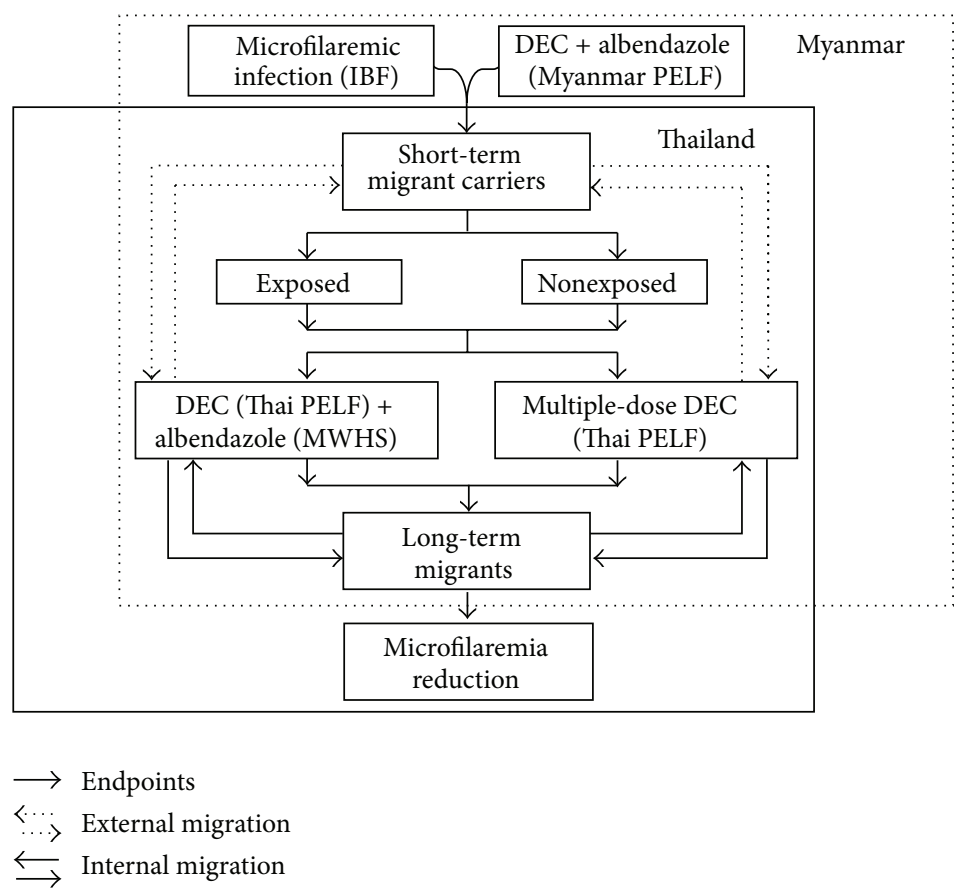

FIGURE 7: Chain migration model of MMWs. Cross-border microfilaremic MMWs who may or may not be treated with MDA 2 drugs in Myanmar become short-term migrant carriers initially after migration to Thailand. If external migration to Myanmar exists, there will be short-term migrant carriers due to discontinuing treatment (nonexposed) with the interventions available in permitted provinces. If internal migration in Thailand exists, there will be long-term migrants that appear to continue treatment (exposed). This long-term migration into Thailand will reflect reduction of microfilaremic infection among the cross-border MMWs.

single-dose administration with $300 \mathrm{mg}$ DEC. Also, a singledose $400 \mathrm{mg}$ albendazole (not part of Thai PELF) is given to MMWs for simultaneous treatment of soil-transmitted helminthiases. This treatment is part of the migrant worker health service (MWHS) program at provincial level, and that can serve as an effective treatment as that used in Myanmar PELF. That is, the increase in compliant number of MMWs in Thailand will increase the coverage of Myanmar PELF. Meanwhile, MMW enrollees living with or without accompanied persons (average 3 persons per enrollee) in the province can access either to community outreach for biannual DEC mass treatment or to supportive DEC treatment via malaria clinics or mobile clinics. Such premeditated treatments can enhance followup and access to health service for those without work permits at provincial level. Although the interventions available at the provincial level in the PELF and MWHS have been considered to ascertain the geographical coverage of expansion services, particularly to the target MMW population, it is necessary for the implementers at all levels to better understand favorable factors that influence mass treatment coverage and compliance.

As noted earlier, it is hypothesized that the increased risks possibly occur when chain migration-either the internal migration from one workplace to another in Thailand or the external migration between Thai and Myanmar-contributes to MDA scheduling and treatment delay in different settings (Figure 7). Moreover, such treatment-seeking behaviors of MMWs-the central model of the "four As" of health behavioral factors-remain to be largely established whether healthcare services to MMWs are associated with availability of drugs and healthcare providers, accessibility of health services, affordability of health insurance and other costs of healthcare, and acceptability of health services, healthcare providers, and excessive bureaucracy of Thai/Myanmar PELF. Until now, little is known about movements of MMWs, and key factors for their treatment-seeking behaviors have never been fully established to increase the consumption of available drugs in different settings and, vice versa, to figure out what factors exert the parasite fitness in those migrant carriers predisposed to the certain MDA 2-drug regimen (DEC plus albendazole). In this paper, we demonstrate that chain migration is a public health challenge to Thai/Myanmar PELF (Figure 7). Short-term migrants including IBF carriers-whether exposed to MDA 2-drug regimen (DEC plus albendazole) implemented in Myanmar-can have chance to receive either one of $300 \mathrm{mg}$ DEC (Thai PELF) plus $400 \mathrm{mg}$ albendazole (MWHS) and multiple-dose DEC (Thai PELF) in the provinces where projecting long-term migration for job applications and, eventually, the IBF can be domestically arrested as the result of microfilaremia reduction. Although the Thai PELF implementing multipledose DEC regimen governs the "four As" factors-enabling reduction of potential "barrier" for adequate free-of-charge treatment in the IBF control efforts, a multiple-dose DEC inaccessibility-a status of MMWs who neither access MDA with $300 \mathrm{mg}$ DEC nor are reluctant to treatment during the stay in permitted province, this undesirable outcome of crossborder MMWs remains unclear. Here, we demonstrate the 
TABLE 1: Univariate analysis of significant individual risk factors associated with multiple-dose DEC inaccessibility between two working groups of cross-border Myanmar migrants (chain migration model).

\begin{tabular}{|c|c|c|c|c|c|c|c|c|c|c|}
\hline \multirow{2}{*}{ Variable } & \multirow{2}{*}{$n(904)$} & \multicolumn{4}{|c|}{ Ranong, 2002-2003 } & \multirow{2}{*}{$n(1,610)$} & \multicolumn{4}{|c|}{ Phangnga, 2004-2006 } \\
\hline & & $\%$ DEC inaccessibility & OR & $95 \% \mathrm{CI}$ & $P$ value & & $\%$ DEC inaccessibility & OR & $95 \% \mathrm{CI}$ & $P$ value \\
\hline \multicolumn{11}{|l|}{ Gender } \\
\hline Male & 553 & 49.7 & 1 & & & 1067 & 42.6 & 1 & & \\
\hline Female & 351 & 56.4 & 1.3 & $1.0-1.7$ & 0.05 & 543 & 48.4 & 1.3 & $1.0-1.6$ & 0.027 \\
\hline \multicolumn{11}{|l|}{ Age group (yr) } \\
\hline$\geq 25$ & 560 & 44.8 & 1 & & & 1106 & 42.9 & 1 & & \\
\hline$<25$ & 344 & 64.5 & 2.2 & $1.7-3.0$ & $<0.001$ & 504 & 48.2 & 1.2 & $1.0-1.5$ & 0.049 \\
\hline \multicolumn{11}{|l|}{ Marital status } \\
\hline Living with a partner & 580 & 45.2 & 1 & & & 1094 & 42.8 & 1 & & \\
\hline $\begin{array}{l}\text { Single/widowed/ } \\
\text { divorced/separated }\end{array}$ & 324 & 65.1 & 2.3 & $1.7-3.0$ & $<0.001$ & 516 & 48.4 & 1.3 & $1.0-1.6$ & 0.033 \\
\hline \multicolumn{11}{|l|}{ Migration pattern } \\
\hline Long-term migration & 643 & 38.4 & 1 & & & 977 & 32.3 & 1 & & \\
\hline Short-term migration & 261 & 86.6 & 10.4 & $7.0-15.3$ & $<0.001$ & 633 & 63.5 & 3.6 & $3.0-4.5$ & $<0.001$ \\
\hline \multicolumn{11}{|l|}{ Occupation } \\
\hline Industry & 447 & 38.3 & 1 & & & 512 & 38.9 & 1 & & \\
\hline Agriculture & 457 & 66.1 & 3.1 & $2.4-4.1$ & $<0.001$ & 1098 & 47.3 & 1.4 & $1.1-1.8$ & 0.002 \\
\hline
\end{tabular}

univariate analysis of the factors associated with low access to multiple-dose MDA with 300 mg DEC using a chain migration model (Figure 7). The significant sociodemographic factors were analyzed using the cross-border MMWs from two data sets between 2002-2003 preimplementation and 20042006 implementation phases of Thai PELF (Table 1). Risk factors associated with multiple-dose DEC inaccessibility $(P<0.05)$ included female, $<25$ years of age, marital status (single), short-term migration, and occupation (agricultural practices). Education level and income were considered significant economic aspects for chain migration, both internal and external, as they also yielded confounding factors which we could not marginalize their spatial heterogeneity in temporary employment opportunities and treatment-seeking behaviors in both study areas of Ranong and Phangnga.

\section{Perspective on Population Migration and Parasite Fitness}

Although there is a dramatically marked decline in LF in Thailand after the effective implementation of Thai PELF, the PCT can interrupt cyclic transmission between infected individuals and mosquito vectors in transmission areas with high degree of MDA 2-drug coverage. Meanwhile, Myanmar PELF has been facing major constraints such as logistic supply and geographical coverage of annual MDA 2 drugs, cross-border migrations of MMWs, and border crossings of local border people between Thailand and Myanmar. These constraints may delude the effectiveness of the implementation of Myanmar PELF, and, accordingly, there is need for the continuation of MDA 2 drugs to cover all eligible target population as well as postgenomic MDA monitoring and evaluation of the parasites in humans and mosquitoes (Figure 8) [3]. The population migration that is the link to the adaptation of the $W$. bancrofti population in complex epidemiological settings of either BBF or IBF is a topic of interest in the SEA region. Thus far, $W$. bancrofti has ability to possess the genetic variability $[20,27,28]$, and hence the parasite population in either BBF or IBF settings may have increased or decreased fitness under treatment and vector control. Although the implementation of Thai/Myanmar PELF operates in the absence of vector control, the MDA 2 drugs may not be only the factor that shapes the parasite population under suppression of Thai and Myanmar PELFs. If the positive selection of $W$. bancrofti occurs with gradual development of resistance to MDA 2 drugs, unbridled transmission of such the $W$. bancrofti population in either BBF or IBF settings will challenge Thai/Myanmar PELF.

The genetically determined resistance to anthelmintic drugs in veterinary nematode parasites can undergo under selective pressure of benzimidazole compounds in a way that albendazole resistance mechanism involves one of two single amino acid substitutions from phenylalanine to tyrosine in parasite $\beta$-tubulin at codon 167 or 200 [3, 19, 24, 29-31]. Albendazole acts as a board-spectrum anthelmintic drug. The drug as an effective benzimidazole compound is used in a single-dose combination of DEC to treat LF patients, as part of the PELF. In this regard, the benzimidazole susceptibility of $W$. bancrofti population in either BBF or IBF settings remains to be established whether the active $W$. bancrofti infections carry the allele frequencies involved in benzimidazole (BZ) susceptibility or resistance (Figure 8 ). If the positive selection 


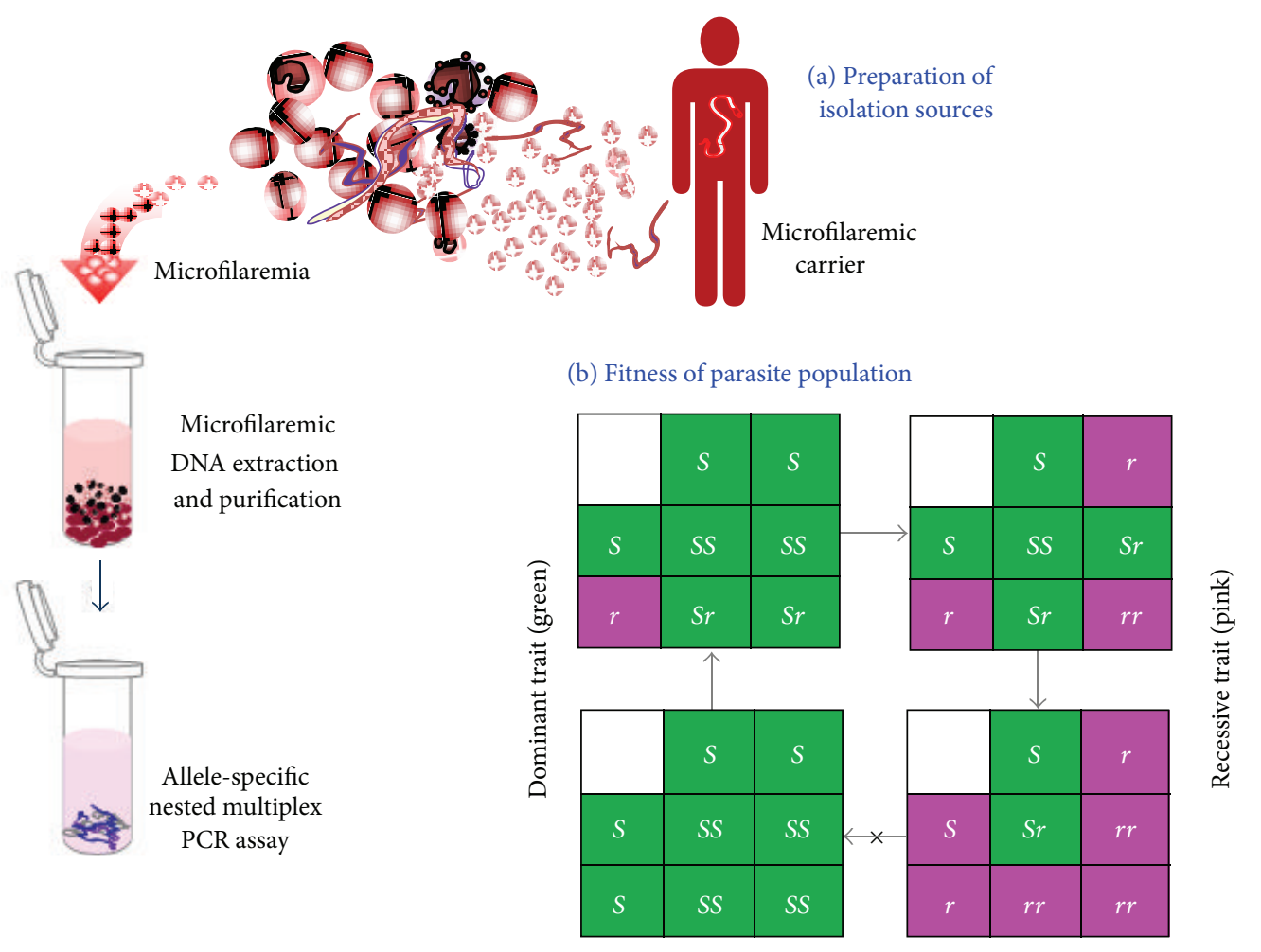

(c) Homozygous BZ-susceptible (SS) isolates

Primer set used: forward primer, BT121 + BT121R Reverse primer, BT122

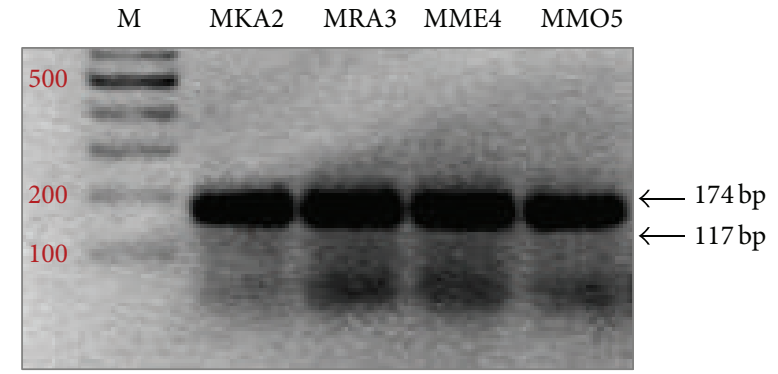

Forward primer, BT121

Reverse primer $\mathrm{BT} 122 \mathrm{~S}+\mathrm{BT} 122$

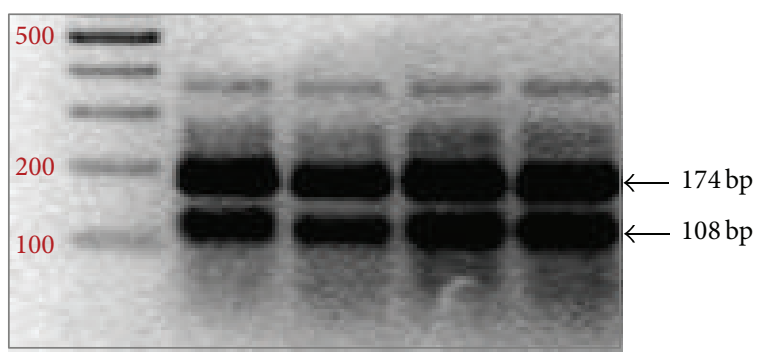

FIGURE 8: Detection and identification of benzimidazole susceptibility and resistance in $W$. bancrofti population by allele-specific nested multiplex PCR assay. (a) IBF microfilaremic carriers used in testing a hypothesis that W. bancrofti parasite population harbor benzimidazole susceptible phenotype although the adaptation of parasite population is influenced by chain migration of MMWs over a space and time as described in Figure 7. (b) Fitness of W. bancrofti parasite population could yield descendants of parasites with allele frequencies in dynamic cross-border MMWs under suppression of Thai/Myanmar PELF. An individual carrying one dominant and one recessive allele (Sr) is referred to as heterozygous dominant. An individual has two dominant alleles (SS), the condition is referred to as BZ-susceptible homozygous allele; an individual with two recessive alleles $(r r)$ is called BZ-resistant homozygous allele. (c) Allele-specific nested multiplex PCR assay whose amplification conditions have been previously established by Bhumiratana et al. (2010) [19] and Pechgit et al. (2011) [24] employs the primers BT121 and BT122 originally derived from the exon 5 of $W$. bancrofti $\beta$-tubulin gene hypothetically conferring a single-point mutation at codon F200Y. Only the primers BT121R and BT122S are newly developed for this investigation. 
of $W$. bancrofti populations increases the fitness of advantageous alleles of $\beta$-tubulin gene, the genetic inheritance of resistant parasite population is thought to be provoked under the pressure of albendazole but not DEC (Figure 8(b)). If $W$. bancrofti may also have increased fitness of polymorphic loci involved in a balanced selection, the heterogeneity of parasite population will decrease fitness of albendazole resistance in the population because the parasites with resistant allele of $\beta$-tubulin gene are not selected by pressure of DEC and thereby the parasites will have homozygous allele frequencies (SS) (Figure 8(b)). In this paper, we first demonstrate the feasibility of using advanced PCR detection method as a monitoring tool for assessing the impacts of MDA 2 drugs on the $W$. bancrofti population in postgenomic MDA evaluation in addition to what is recommended by WHO.

For instance, the detection and identification of BZsusceptible and-resistant alleles of $W$. bancrofti $\beta$-tubulin gene have been performed with allele-specific nested multiplex PCR using some isolation sources of IBF cases, namely, MKA2, MME4, MMO5, and MRA3 isolates originally obtained from microfilaremic MMWs (Figures 8(a) and 3(c)) [30]. The separately nested reactions were performed on amplifications of primary products initially amplified using primers specific for the segment of $W$. bancrofti $\beta$-tubulin gene spanning $3^{\prime}$ partial exon3-intron-exon4-intron-exon5$5^{\prime}$ partial intron sequences $[19,24]$. The nucleotide sequences of this DNA segment of these isolates have been deposited in GenBank genome database under accession nos. GU190725GU190728, respectively. In the secondary reaction testing parasites with BZ-susceptible allele, the expected duplex amplicons are 174 and 108 bp using forward primer BT121 and two reverse primers BT122 and BT122S $5^{\prime}$ ATACAAAGCCTCGTTATCAATGCAGA $3^{\prime}$. The $108 \mathrm{bp}$ fragment is the homozygous $S S$ allele. In the secondary reaction testing parasites with BZ-resistant allele, the expected duplex amplicons are 174 and $117 \mathrm{bp}$ using two forward primers BT121 and BT121R 5' CTAGTTGAAAACACTGACGAAACTTA $3^{\prime}$ and reverse primer BT122. The $117 \mathrm{bp}$ fragment is the homozygous $r r$ allele. As for the heterozygous $\mathrm{Sr}$ allele, consistently positive amplification could yield expected duplex amplicons in both reactions. Here, we first demonstrated that the IBF carry the parasites with homozygous BZ-susceptible (SS) allele. This might suggest that the IBF will carry homozygous allele frequencies $(S S)$ greater than heterozygous allele frequencies $(\mathrm{Sr})$ and homozygous allele associated with albendazole resistance whether the IBF is genetically predisposed to the MDA 2-drug regimen (DEC plus albendazole) implemented in Myanmar. However, further investigations are required to analyze $W$. bancrofti parasite populations with wild-type, namely, mutant allele frequencies so as to whether they are associated with pressure of the MDA 2 drugs and other vulnerability observed in the IBF or BBF settings.

\section{Acknowledgments}

The authors acknowledge Dr. Pisit Yongyuth, Dr. Prapassorn Pechgit, Dr. Ronald A. Markwardt, and Dr. Jinrapa Pothikasikorn for providing helpful information and suggestions.

\section{References}

[1] WHO, "Global programme to eliminate lymphatic filariasis," Weekly Epidemiological Record, vol. 83, no. 37, pp. 333-341, 2008.

[2] WHO, Regional Strategic Plan for Elimination of Lymphatic Filariasis (2000-2004), Mimeographed document SEA/FIL/ 28.corr. 1., World Health Organization; South-East Asia Regional Office, New Delhi, India, 2001.

[3] A. Bhumiratana, A. Intarapuk, D. Sangthong, S. Koyadun, P. Pechgit, and J. Pothikasikorn, "Molecular diagnosis and monitoring of benzimidazole susceptibility of human filariids," in Current Topics in Tropical Medicine, A. J. Rodriguez-Morales, Ed., pp. 397-424, 2012.

[4] E. A. Ottesen, B. O. L. Duke, M. Karam, and K. Behbehani, "Strategies and tools for the control/elimination of lymphatic filariasis," Bulletin of the World Health Organization, vol. 75, no. 6, pp. 491-503, 1997.

[5] D. Kyelem, G. Biswas, M. J. Bockarie et al., "Determinants of success in national programs to eliminate lymphatic filariasis: a perspective identifying essential elements and research needs," American Journal of Tropical Medicine and Hygiene, vol. 79, no. 4, pp. 480-484, 2008.

[6] J. O. Gyapong, V. Kumaraswami, G. Biswas, and E. A. Ottesen, "Treatment strategies underpinning the global programme to eliminate lymphatic filariasis," Expert Opinion on Pharmacotherapy, vol. 6, no. 2, pp. 179-200, 2005.

[7] M. J. Bockarie, M. J. Taylor, and J. O. Gyapong, "Current practices in the management of lymphatic filariasis," Expert Review of Anti-Infectious Therapy, vol. 7, no. 5, pp. 595-605, 2009.

[8] WHO, Progress Report 2000-2009 and Strategic Plan 20102020 of the Global Programme to Eliminate Lymphatic Filariasis: Halfway Towards Eliminating Lymphatic Filariasis, Mimeographed document WHO/HTM/NTD/PCT/2010. 6, World Health Organization, Geneva, Switzerland, 2010.

[9] WHO, Regional Programme Review Group (RPRG) for Elimi-nation of Lymphatic Filariasis in South-East Asia Region, Mimeographed document SEA-CD-211, South-East Asia Regional Office, Report of the Seventh Meeting, Jakarta, Indonesia; World Health Organization, New Delhi, India, 2010.

[10] WHO, Guidelines for Certifying Lymphatic Filariasis Elimination (Including Discussion of Critical Issues and Rationale), Following from InFormal Consultation on Epidemiologic Approaches to Lymphatic Filariasis Elimination: Initial Assessment, Monitoring, and Certification, Atlanta, Georgia, USA 2-4 September 1998, Mimeographed document WHO/FIL/99/197, World Health Organization, Geneva, Switzerland, 1999.

[11] WHO, Informal Consultation on Epidemiologic Approaches to Lymphatic Filariasis Elimination: Initial Assessment, Monitoring, and Certification, Atlanta, Georgia, USA 2-4 September 1998, Mimeographed document WHO/FIL/99. 195, World Health Organization, Geneva, Switzerland, 1999.

[12] WHO, "Transmission assessment surveys in the Global Programme to Eliminate Lymphatic Filariasis: WHO position statement," Weekly Epidemiological Record, vol. 87, no. 48, pp. 478-482, 2012.

[13] A. Bhumiratana, S. Koyadun, S. Suvannadabba et al., "Field trial of the ICT filariasis for diagnosis of Wuchereria bancrofti infections in an endemic population of Thailand," Southeast Asian Journal of Tropical Medicine and Public Health, vol. 30, no. 3, pp. 562-568, 1999.

[14] A. Bhumiratana, B. Wattanakull, S. Koyadun, S. Suvannadabba, J. Rojanapremsuk, and W. Tantiwattanasup, "Relationship 
between male hydrocele and infection prevalences in clustered communities with uncertain transmission of Wuchereria bancrofti on the Thailand-Myanmar border," Southeast Asian Journal of Tropical Medicine and Public Health, vol. 33, no. 1, pp. 7-17, 2002.

[15] A. Bhumiratana, S. Koyadun, P. Satitvipawee, M. Srisuphanunt, N. Limpairojn, and G. Gaewchaiyo, "Border and imported bancroftian filariases: baseline seroprevalence in sentinel populations exposed to infections with Wuchereria bancrofti and concomitant HIV at the start of diethylcarbamazine mass treatment in Thailand," Southeast Asian Journal of Tropical Medicine and Public Health, vol. 36, no. 2, pp. 390-407, 2005.

[16] C. Apiwathnasorn, Y. Samung, S. Prummongkol, A. Asavanich, N. Komalamisra, and P. Mccall, "Bionomics studies of Mansonia mosquitoes inhabiting the peat swamp forest," Southeast Asian Journal of Tropical Medicine and Public Health, vol. 37, no. 2, pp. 272-278, 2006.

[17] S. Koyadun and A. Bhumiratana, "Surveillance of imported bancroftian filariasis after two-year multiple-dose diethylcarbamazine treatment," Southeast Asian Journal of Tropical Medicine and Public Health, vol. 36, no. 4, pp. 822-831, 2005.

[18] A. Bhumiratana, C. Siriaut, S. Koyadun, K. Anurat, and P. Satitvipawee, "Evaluation of a single oral dose of diethylcarbamazine $300 \mathrm{mg}$ as provocative test and simultaneous treatment in Myanmar migrant workers with Wuchereria bancrofti infection in Thailand," Southeast Asian Journal of Tropical Medicine and Public Health, vol. 35, no. 3, pp. 591-598, 2004.

[19] A. Bhumiratana, P. Pechgit, S. Koyadun, C. Siriaut, and P. Yongyuth, "Imported bancroftian filariasis: diethylcarbamazine response and benzimidazole susceptibility of Wuchereria bancrofti in dynamic cross-border migrant population targeted by the National Program to Eliminate Lymphatic Filariasis in South Thailand," Acta Tropica, vol. 113, no. 2, pp. 121-128, 2010.

[20] S. Nuchprayoon, A. Junpee, and Y. Poovorawan, "Random amplified polymorphic DNA (RAPD) for differentiation between Thai and Myanmar strains of Wuchereria bancrofti," Filaria Journal, vol. 6, article 6, 8 pages, 2007.

[21] P. Yongyuth, S. Koyadun, N. Jaturabundit, A. Sampuch, and A. Bhumiratana, "Efficacy of a single-dose treatment with $300 \mathrm{mg}$ diethylcarbamazine and a combination of $400 \mathrm{mg}$ albendazole in reduction of Wuchereria bancrofti antigenemia and concomitant geohelminths in Myanmar migrants in Southern Thailand," Journal of the Medical Association of Thailand, vol. 89, no. 8, pp. 1237-1248, 2006.

[22] S. Nuchprayoon, C. Porksakorn, A. Junpee, V. Samprasert, and Y. Poovorawan, "Comparative assessment of an Og4C3 ELISA and ICT Filariasis Test: a study of Myanmar migrants in Thailand," Asian Pacific Journal of Allergy and Immunology, vol. 21, no. 4, pp. 253-257, 2003.

[23] J. Pothikasikorn, M. J. Bangs, R. Boonplueang, and T. Chareonviriyaphap, "Susceptibility of various mosquitoes of Thailand to nocturnal subperiodic Wuchereria bancrofti," Journal of Vector Ecology, vol. 33, no. 2, pp. 313-320, 2008.

[24] P. Pechgit, A. Intarapuk, D. Pinyoowong, and A. Bhumiratana, "Touchdown-touchup nested PCR for low-copy gene detection of benzimidazole-susceptible Wuchereria bancrofti with a Wolbachia endosymbiont imported by migrant carriers," Experimental Parasitology, vol. 127, no. 2, pp. 559-568, 2011.

[25] A. Bhumiratana, P. Sorosjinda-Nunthawarasilp, W. Kaewwaen, P. Maneekan, and S. Pimnon, "Malaria-associated rubber plantations in Thailand," Travel Medicine and Infectious Disease, 2012.
[26] W. Satimai, C. Jiraamonnimit, S. Thammapalo et al., "The impact of a national program to eliminate lymphatic filariasis in selected Myanmar immigrant communities in Bangkok and Ranong Province, Thailand," Southeast Asian Journal of Tropical Medicine and Public Health, vol. 42, no. 5, pp. 1054-1064, 2011.

[27] N. Pradeep Kumar, K. P. Patra, S. L. Hoti, and P. K. Das, "Genetic variability of the human filarial parasite, Wuchereria bancroft $i$ in South India," Acta Tropica, vol. 82, no. 1, pp. 67-76, 2002.

[28] S. L. Hoti, R. Thangadurai, R. Dhamodharan, and P. K. Das, "Genetic heterogeneity of Wuchereria bancrofti populations at spatially hierarchical levels in Pondicherry and surrounding areas, south India," Infection, Genetics and Evolution, vol. 8, no. 5, pp. 644-652, 2008.

[29] S. L. Hoti, R. Dhamodharan, K. Subramaniyan, and P. K. Das, "An allele specific PCR assay for screening for drug resistance among Wuchereria bancrofti populations in India," Indian Journal of Medical Research, vol. 130, no. 2, pp. 193-199, 2009.

[30] A. E. Schwab, D. A. Boakye, D. Kyelem, and R. K. Prichard, "Detection of benzimidazole resistance-associated mutations in the filarial nematode Wuchereria bancrofti and evidence for selection by albendazole and ivermectin combination treatment," American Journal of Tropical Medicine and Hygiene, vol. 73, no. 2, pp. 234-238, 2005.

[31] B. J. Fennell, J. A. Naughton, J. Barlow et al., "Microtubules as antiparasitic drug targets," Expert Opinion on Drug Discovery, vol. 3, no. 5, pp. 501-518, 2008. 


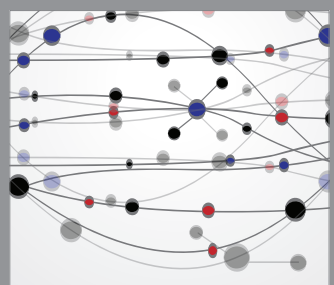

The Scientific World Journal
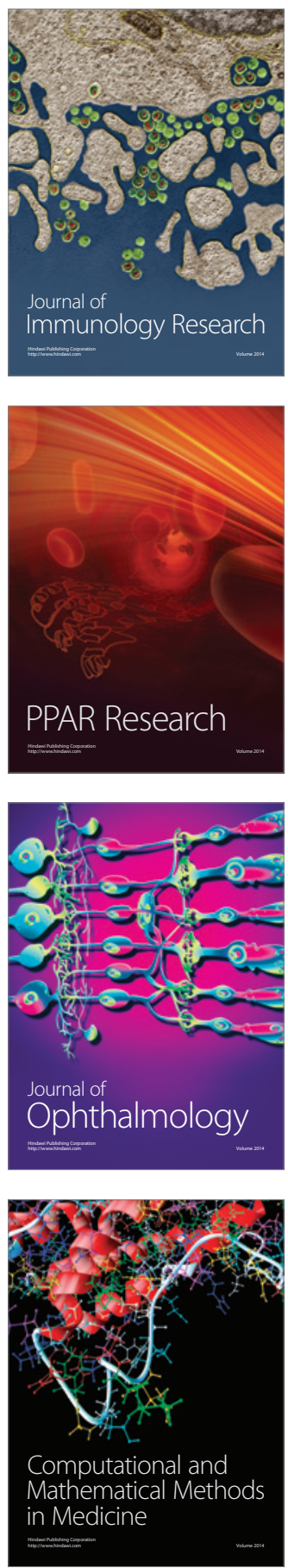

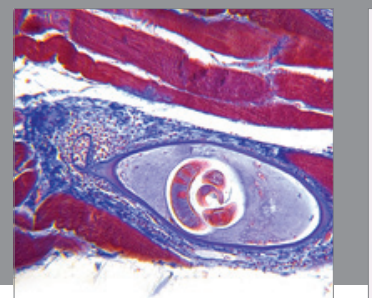

Gastroenterology

Research and Practice
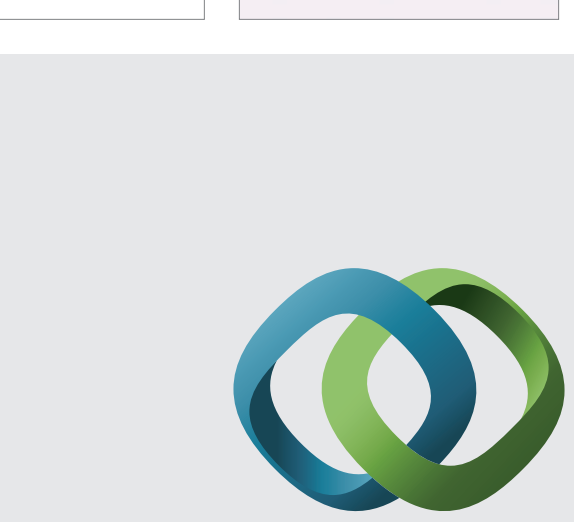

\section{Hindawi}

Submit your manuscripts at

http://www.hindawi.com
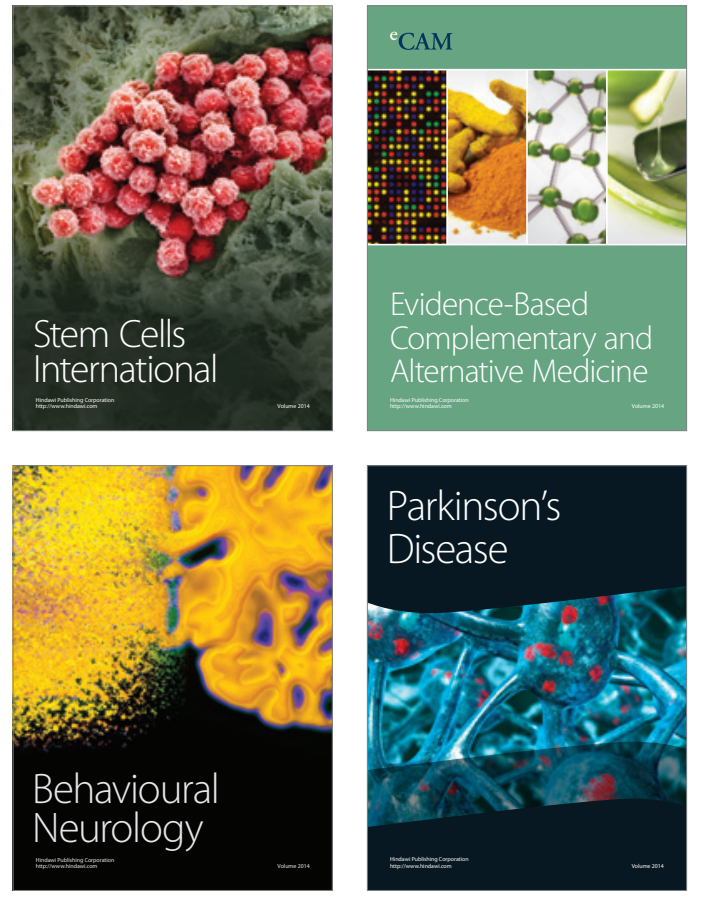
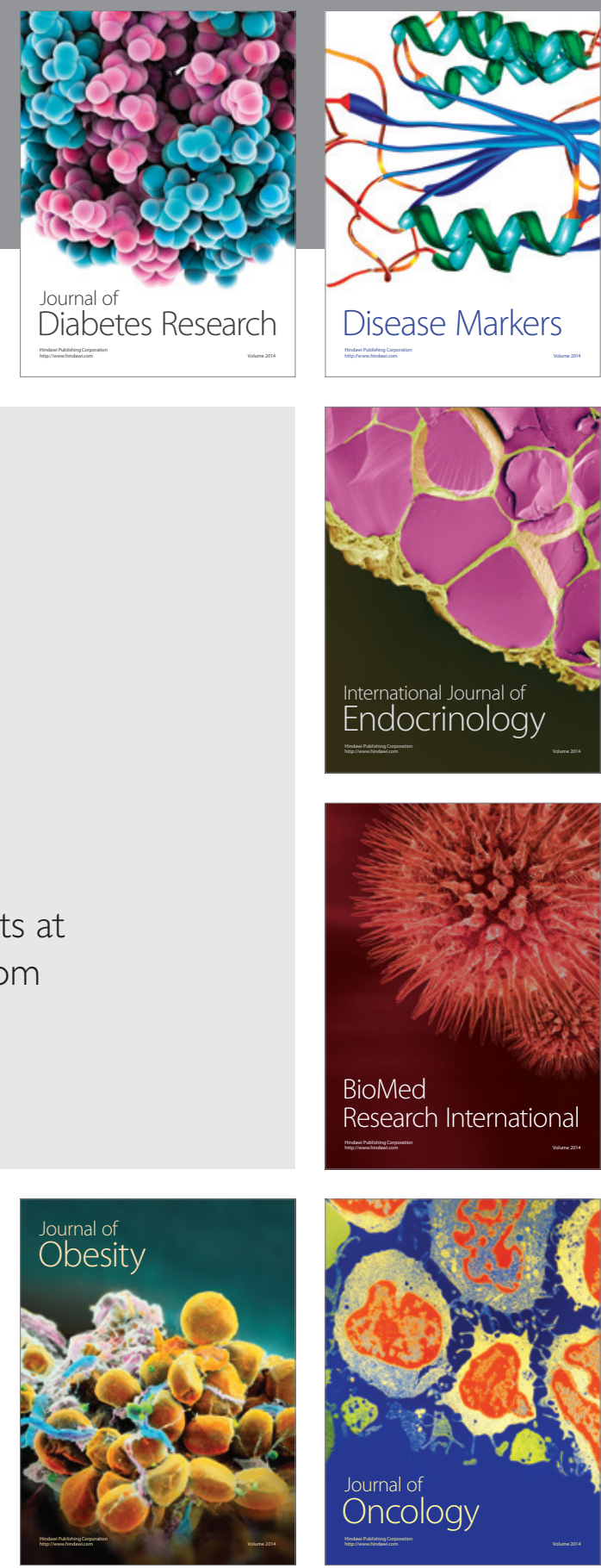

Disease Markers
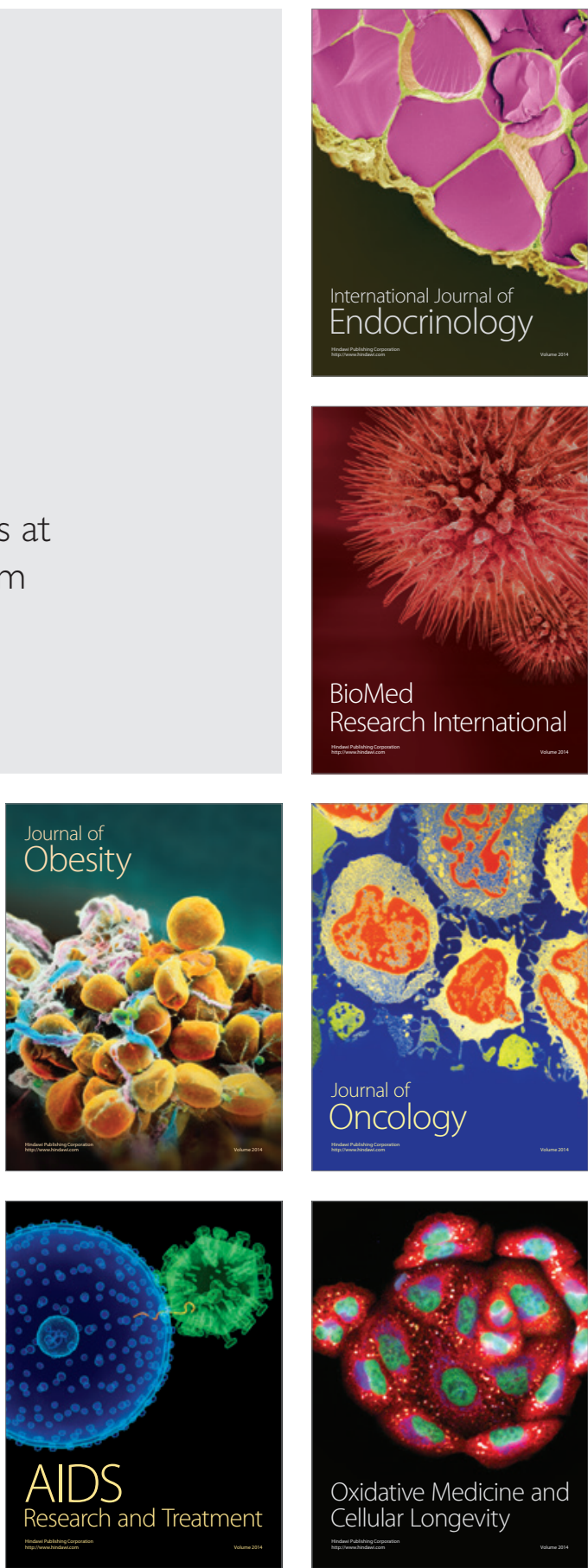